\title{
Efeito da fisioterapia convencional e do feedback eletromiográfico associados ao treino de tarefas específicas na recuperação motora de membro superior após acidente vascular encefálico
}

\author{
Efeito da fisioterapia convencional e do feedback eletromiográfico \\ associados ao treino de tarefas específicas na recuperação motora de \\ membro superior após acidente vascular encefálico
}

\author{
R.C. Araújo, M.P. Barbosa
}

ARTIGO ORIGINAL | ORIGINAL ARTICLE

\begin{abstract}
RESUMO
O objetivo do presente estudo foi avaliar os efeitos da fisioterapia convencional e do feedback eletromiográfico (EMG) associados ao treino de tarefas específicas na recuperação da função motora do membro superior de pacientes com hemiparesia em consequência do acidente vascular encefálico. Doze pacientes foram avaliados e divididos de forma aleatória em dois grupos: Grupo 1 - Fisioterapia convencional, Grupo 2 -Feedback EMG. Todos os pacientes receberam três sessões semanais de 50 minutos de duração, por oito semanas. Foram avaliados o comprometimento motor, funcionalidade e espasticidade, por meio do teste Fugl-Meyer (FMA), TEMPA e Escala Modificada de Ashworth, respectivamente. Os resultados mostraram que em ambos os grupos não houve alteração da espasticidade e que ocorreu melhora significativa nos valores de FMA, após os programas de reabilitação. Em relação aos escores do TEMPA, somente o grupo 2 apresentou ganhos significativos. A comparação dos ganhos obtidos na escala modificada de Ashworth e FMA não revelou diferenças entre os grupos. No entanto, o grupo 2 apresentou ganhos superiores ao grupo 1 em duas tarefas do teste TEMPA. Os resultados sugerem que o treino funcional utilizando o feedback EMG pode ter efeitos positivos na melhora da função motora do braço, no entanto, devido às pequenas diferenças encontradas entre os grupos, concluiu-se que o feedback EMG não foi superior à outra forma de tratamento.

Palavras-chave: eletromiografia, reabilitação, acidente vascular encefálico
\end{abstract}

ABSTRACT

The purpose of the present study was to evaluate the effects of conventional physical therapy and task specific training associated with electromyographic (EMG) feedback to recover upper limb motor function in patients with hemiparesis caused by stroke. Twelve patients were evaluated and then randomly assigned to either of two groups: Group 1 - Conventional physiotherapy; Group 2 - EMG feedback. Patients attended three weekly 50-min sessions, for eight weeks. The evaluated aspects were motor impairment, motor function, and spasticity, using the Fugl-Meyer Assessment (FMA) test, TEMPA, and the Modified Ashworth Scale (MAS), respectively. Results showed that, in both groups, there were no changes in spasticity in the groups, but there was a significant improvement in FMA values after the rehabilitation programs. Regarding TEMPA scores, only Group 2 presented significant gains. The comparison of the MAS and FMA gains showed no differences between groups. However, group 2 showed greater gains in the two tasks of TEMPA. The results suggest that functional training using EMG feedback can have positive results in improving arm function. However, due to small differences between groups, concluded that EMG/Biofeedback therapy was not superior to other form of treatment.

Keywords: electromyography, rehabilitation, stroke

Submetido: 24.03.2012 | Aceite: 04.0.2012

Rodrigo Cappato de Araújo. Universidade de Pernambuco - UPE, Brasil.

Marcos Pinotti Barbosa. Universidade Federal de Minas Gerais - UFMG, Brasil.

Endereço para correspondência: Rodrigo Cappato de Araújo, Universidade de Pernambuco, Campus Petrolina,

Departamento de Fisioterapia. BR 203 Km 2 S/N, Vila Eduardo, 56300-000 - Petrolina, PE - Brasil.

E-mail: rodrigocappato@yahoo.com.br 
Nos últimos anos, tem ocorrido um significante aumento da expectativa de vida, com consequente aumento da população mundial de idosos. No entanto, concomitante ao aumento da expectativa de vida, observa-se um aumento das co-morbidades relacionadas ao envelhecimento. Entre estas co-morbidades, destaca-se o acidente vascular encefálico (AVE). O AVE é resultado de um dano celular, devido à isquemia ou hemorragia no tecido encefálico, podendo causar déficits de várias funções sensório-motoras (Rathore, Hinn, Cooper, Tyroler, \& Rosamond, 2002).

Estudos recentes relataram uma prevalência de 200 a 300 novos casos de AVE em cada 100.000 habitantes por ano, nos Estados Unidos e na Europa (Muntner, Garret, Klag, \& Coresh, 2002), contabilizando atualmente no mundo um grande número de indivíduos que sobrevivem ao AVE e que convivem com alguma incapacidade funcional (Feigin, 2005).

Convencionalmente, programas de reabilitação têm sido conduzidos por fisioterapeutas em hospitais ou centros de reabilitação ao longo da história. Estes programas são caracterizados pela aplicação de técnicas e exercícios terapêuticos que estimulam o reaprendizado motor e que têm por objetivo aumentar a independência funcional (Levy, Nichols, Schmalbrock, Keller, \& Chakeres, 2001; Liepert et al., 2000; Schaechter et al., 2002). No entanto, cerca de $30-66 \%$ dos pacientes avaliados seis meses após o AVE não apresentaram função do membro superior do lado afetado e somente $5 \%$ demonstraram completa recuperação das atividades funcionais (Gowland, 1982; Kwakkel, Kollen, \& Wagenaar, 1999). Diante dos resultados limitados no processo de reabilitação da função motora do membro superior, pesquisas recentes têm investigado a implementação de outras técnicas terapêuticas, dentre essas se destaca o feedback eletromiográfico (feedback EMG).

O feedback EMG pode ser descrito como um método que possibilita a captação dos potenciais de ação do músculo, por meio de eletrodos de superfície, e a conversão desses potenciais em informações visuais e/ou sonoras. Essas informações permitem ao paciente controlar e regular a atividade muscular (Hurrel, 1980; Moreland \& Thomson, 1994).

Em geral, se encontra na literatura resultados positivos do uso do feedback EMG na reabilitação da marcha (Aiello et al., 2005; Bradley et al., 1998) e da função motora do membro superior em pacientes com sequelas do AVE (Armagan, Tascioglu, \& Oner, 2003; Crow, Lincoln, Nouri, \& Weerdt, 1989; Hurd, Pegram, \& Nepomuceno, 1980; Inglis, Donald, Monga, Sproule, \& Young, 1984). Entretanto, revisões sistemáticas recentes mostraram existir controvérsias acerca da efetividade e da superioridade do feedback EMG em relação ao tratamento convencional, no que diz respeito à reabilitação motora do membro superior (Moreland \& Thomson, 1994). Segundo os autores, os resultados presentes na literatura devem ser interpretados com cautela, pois vieses metodológicos referentes à duração da intervenção, bem como os métodos utilizados para avaliação, podem ter influenciado os resultados.

Outro problema importante que estaria relacionado à dificuldade de se evidenciar os efeitos benéficos do uso do feedback EMG no processo de reabilitação motora é o modo de aplicação da técnica. Embora seja amplamente reconhecida a importância da inserção de atividades funcionais orientadas no programa de reabilitação, o feedback EMG tem sido frequentemente aplicado em posturas estáticas e em movimentos que não fazem parte de uma atividade funcional (Jonsdottir et al., 2010). Segundo os mesmos autores, a associação do feedback EMG à tarefas funcionais orientadas poderia maximizar os resultados da terapia e facilitar o processo de aprendizagem motora. Considerando estas informações, o objetivo deste estudo foi avaliar o treinamento funcional associado ao feedback EMG e compará-lo com um programa fisioterapêutico, na reabilitação motora do membro 
superior de pacientes vítimas do AVE.

\section{MÉTODO}

\section{Amostra}

O estudo foi realizado com doze pacientes de ambos os sexos (10 homens e 2 mulheres) e que apresentavam com diagnóstico clínico de AVE primário de causa isquêmica ou hemorrágica há pelo menos três meses. Foram incluídos pacientes destros, que não apresentaram déficits de compreensão e que apresentaram comprometimento motor do membro superior direito. Foram excluídos os pacientes que apresentaram sequelas motoras bilaterais ou no hemicorpo esquerdo e que tivessem histórico de dois ou mais acidentes vasculares encefálicos. Voluntários que apresentaram espasticidade severa (valores superiores a três na escala modificada de Ashworth) e que não tenham conseguido realizar qualquer uma das tarefas dos teste TEMPA também foram excluídos da amostra. Esse estudo foi aprovado pelo Comitê de Ética em Pesquisa da Universidade de Pernambuco, registro CEP-UPE 041/09.

\section{Instrumentos e Procedimentos}

Inicialmente, foi realizada uma avaliação física, na qual foram colhidos os dados pessoais e antropométricos dos sujeitos, além do histórico e questões relacionadas ao AVE. Em seguida os voluntários foram submetidos a uma avaliação funcional, na qual foi realizado o teste de Fugl-Meyer (FMA) (Fulg-Meyer, Jaasko, Leyman, Olsson, \& Steglind, 1975) para avaliação do comprometimento motor do membro superior. A escala de avaliação motora de membro superior do teste FMA, utilizada neste estudo, inclui um sistema de pontuação numérica acumulativa que avalia os movimentos de ombro, cotovelo, antebraço, punho e mão. O escore total da escala de membro superior varia entre 0 e 66 pontos, sendo que escores entre 50 a 65 pontos significam comprometimento motor leve, entre 30 e 49 pontos refletem comprometimento moderado e valores abaixo de 30 demonstram comprometimento motor grave (Michaelsen \& Levin, 2004; Michaelsen, Dannenbaum, \& Levin, 2006). A versão brasileira do FMA utilizada nesse estudo apresenta excelente confiabilidade (ICC > 0.98) para o escore total de avaliação do membro superior (Michaelsen, Rocha, Knabben, Rodrigues, \& Fernandes, 2011).

A avaliação da espasticidade muscular dos flexores de cotovelo, punho e dedos foi realizada por meio da Escada Modificada de Ashworth, que possui uma escala numérica variando de 0 a 5 pontos (Brashear et al., 2002). A funcionalidade e destreza manual foram avaliadas por meio do teste TEMPA (Test D'Évaluation dês Membres Supérieurs de Personnes Âgeés) versão brasileira (Michaelsen, Natalio, Silva, \& Pagnussat, 2008). O TEMPA é um teste para avaliação do desempenho do membro superior e é composto por quatro tarefas bilaterais (Abrir um pote e tirar uma colher cheia de café; Destrancar uma fechadura, pegar e abrir um pote contendo pílulas; Escrever um envelope e colar um selo; Embaralhar e distribuir cartas de um baralho) e quatro tarefas unilaterais (Alcançar e mover um pote; Manusear uma jarra e servir água em um copo; Manusear moedas; Manusear pequenos objetos).

Os escores obtidos são baseados em três critérios: velocidade de execução, graduação funcional e análise das tarefas. $\mathrm{Na}$ avaliação da velocidade, a tarefa é cronometrada desde o início até o seu término. A graduação funcional refere-se à autonomia do sujeito durante a realização de cada tarefa, sendo graduada em quatro níveis: (0) tarefa completada com sucesso sem hesitação e dificuldade, (-1) tarefa completada com alguma dificuldade, (-2) tarefa parcialmente executada ou certas etapas são realizadas com dificuldade e (-3) não consegue completar a tarefa mesmo com auxílio.

A análise das tarefas avalia as dificuldades encontradas pelos sujeitos de acordo com cinco itens relacionados com as habilidade sensório-motoras: força, amplitude de movimento, 
precisão de movimentos amplos, precisão de movimentos finos e preensão.

O escore da graduação funcional é determinado pela adição dos escores obtidos nas tarefas unilaterais à direita $(0 \mathrm{a}-12)$, à esquerda (0 a -12) e nas tarefas bilaterais (0 a -12), totalizando escores entre 0 e -36 . Realiza-se também a avaliação das cinco dimensões da sessão análise das tarefas. Considerando que 'precisão dos movimentos finos' não é mensurada nas tarefas 'pegar e transportar um pote' e 'pegar uma jarra e servir água' e 'força' não é avaliada nas tarefas 'escrever e colar um selo', 'pegar e transportar objetos pequenos', 'manusear moedas' e 'embaralhar cartas', a pontuação da análise de tarefas pode variar de 0 a -150 .

O escore total $(0$ a -186) representa a soma da graduação funcional e da análise das tarefas. Respeitando as dimensões avaliadas em cada tarefa, é possível se obter os seguintes escores: Tarefa 1 (Abrir um pote e tirar uma colher cheia de café): 0 a -18; Tarefa 2 (Destrancar uma fechadura, pegar e abrir um pote contendo pílulas): 0 a -18; Tarefa 3 (Escrever um envelope e colar um selo): 0 a -15; Tarefa 4 (Embaralhar e distribuir cartas de um baralho): 0 a -15; Tarefa 5 (Alcançar e mover um pote): 0 a -30;

Tarefa 6 (Manusear uma jarra e servir água em um copo): 0 a -30; Tarefa 7 (Manusear moedas): 0 a -30; Tarefa 8 (Manusear pequenos objetos): 0 a -30 . As tarefas unilaterais apresentam escores variando de 0 a -30 , pois nesse caso somam-se os escores obtidos na avaliação dos dois membros. Entretanto, neste estudo não foi considerado o tempo de execução das tarefas unilaterais realizadas com o membro sadio. Essa medida foi adotada com o objetivo de evitar possíveis influências do membro sadio na análise do tempo total de execução das tarefas (Platz et al., 2009).

Apesar da escala original propor uma cotação negativa, no qual zero é indicativo de ausência de incapacidade e os valores negativos são indicativos de maior incapacidade, para fins de análise estatística foram utilizados os valores independente do sinal. Desta forma, para este estudo, valores maiores correspondem à maior incapacidade.

Em estudo prévio (Michaelsen et al., 2008) foi demonstrada adequada confiabilidade intra e inter-examinadores (ICC 0.70 - 1.00) dos escores da versão brasileira do TEMPA aplicados em pacientes com déficits motores moderados (FMA < 50).

Após a realização da avaliação funcional, os pacientes foram divididos de forma aleatória em dois grupos: Grupo 1 - seis pacientes (5 homens e 1 mulher) submetidos a um programa de reabilitação fisioterapêutica convencional associada ao treinamento funcional; Grupo 2 seis pacientes (5 homens e 1 mulher) submetidos a um treino funcional associado ao feedback eletromiográfico. Para a alocação dos voluntários em ambos os grupos foi utilizado o programa Winpepi versão 10.8, no qual foi realizado a randomização simples (não-estratificada).

Programa de reabilitação Fisioterapêutica: $\mathrm{O}$ treinamento foi constituído de vinte e quatro sessões, realizadas três vezes por semana durante oito semanas. O programa de reabilitação dos pacientes deste grupo foi conduzido pelo mesmo fisioterapeuta em todas as sessões. Cada sessão teve a duração de 50 minutos e o tratamento aplicado aos pacientes teve como base os princípios do desenvolvimento neuromotor, sendo trabalhado o controle e equilíbrio postural, mobilização escapular, exercícios de descarga de peso para membros superiores e principalmente o treino funcional do hemicorpo comprometido, evoluindo de tarefas simples para padrões de movimentos mais avançados. Em cada sessão de fisioterapia foi realizada aproximadamente 10 minutos de exercícios e orientações de correção da postura de tronco e cintura escapular. Posteriormente, foram treinadas atividades de auto-cuidado (pentear os cabelos, vestir, alimentação e escovar os dentes) e tarefas funcionais (alcance e preensão de diferentes objetos, bem como a movimentação 
desses objetos sobre uma mesa) de membros superiores, nas quais os voluntários precisavam mostrar habilidade de desempenhar tarefas funcionais simples, antes de progredir para padrões funcionais mais avançados. O treino dessas atividades apresentou duração média de 20 minutos, e a para progressão de cada tarefa, foi implementado o aumento do número de repetições e o aumento da massa dos objetos que deveriam ser segurados pelos pacientes. Nos últimos 20 minutos foram realizados várias repetições dos movimentos de flexão e extensão das articulações do cotovelo, punho e mão. Inicialmente foram implementados exercícios ativo-assistido, progredindo para ativo-resistido, conforme evolução de cada paciente.

Programa de reabilitação EMG feedback: Assim como no grupo 1, o protocolo de treinamento com feedback eletromiográfico também foi realizado três vezes por semana durante oito semanas, totalizando 24 sessões de 50 minutos de duração. Para o registro dos sinais miolétricos foram utilizados seis canais do sistema Myosystem BrI (Datahominis Ltda., Uberlândia - Brasil), com aquisição simultânea, aterramento comum a todos os canais, filtros de baixa passagem de $10 \mathrm{HZ}$ a $5 \mathrm{KHz}$, três estágios de amplificação, ganho total de 1000 vezes, impedância dos canais de $10 \mathrm{G} \Omega$ em modo diferencial, 16 bits de faixa de resolução dinâmica, faixa de amplitude de $-10 \mathrm{~V}$ a $+10 \mathrm{Ve}$ frequência de amostragem por canal de $4 \mathrm{KHz}$. Para a visualização e processamento dos sinais foi utilizado o programa Myosystem BrI versão 2.12.

Os sinais mioelétricos dos músculos bíceps braquial, tríceps braquial, flexor radial do carpo, extensor radial do carpo, flexor superficial dos dedos e extensor comum dos dedos foram captados por meio da colocação de eletrodos de superfície simple-diferencial de ganho de 20 vezes, composto por duas barras retangulares paralelas (Datahominis Ltda, UberlândiaBrasil). Antes da colocação dos eletrodos, foram realizadas a tricotomia e a limpeza da pele. Os eletrodos dos músculos bíceps do braço, e tríceps do braço foram posicionados conforme recomendações da European Recommendations for Surface Electromyography do Projeto SENIAN (Hermes et al., 1999). No entanto, os eletrodos do músculo flexor radial do carpo, extensor radial do carpo, flexor superficial dos dedos e extensor comum dos dedos foram posicionados seguindo orientações descritas no estudo de $\mathrm{Hu}$ et al. (2009). A fixação dos eletrodos foi feita com tiras de esparadrapo, permitindo o melhor acoplamento entre as barras de captação e a pele do voluntário. O eletrodo de referência foi posicionado na região do manúbrio esternal, interposto com gel condutor.

Durante cada sessão os voluntários realizaram, por 25 minutos, várias repetições dos movimentos de flexão e extensão das articulações do cotovelo, punho e mão, evoluindo de terapia ativo-assistida para ativa-resistida (resistência manual). Para cada movimento foi definido uma faixa de ativação específica que fosse capaz de disparar o feedback sonoro, e cada voluntário foi orientado a manter o som, sustentando o nível do sinal eletromiográfico. Além disso, os voluntários puderam acompanhar a atividade EMG pela tela do computador, sendo assim utilizado o feedback sonoro simultaneamente ao visual.

$\mathrm{Na}$ segunda metade das sessões, os voluntários foram orientados a realizar tarefas de alcançar e transportar diferentes objetos colocados sobre uma mesa, encorajando-os ao treino dos movimentos de alcance e preensão manual. Para realização dessas tarefas, os voluntários foram posicionados sentados em frente a uma mesa onde se encontravam diferentes objetos que deveriam ser alcançados e transferidos de local. Além disso, sobre a mesa foi posicionado o computador, no qual os voluntários deveriam visualizar a atividade EMG e tentariam coordenar a ação muscular.

Para a adaptação dos pacientes ao sistema, foram realizados várias repetições do movimento de alcance de objetos, nas quais os voluntários deveriam observar a atividade do 
músculo tríceps braquial durante a extensão do cotovelo e a atividade dos músculos extensores de punho e dedos para abertura da mão. Posteriormente, foi solicitado o movimento de preensão manual, no qual deveria ser observado a atividade dos músculos flexores de punho e dedos.

Após adaptação e realização correta das tarefas de alcance e preensão, foi solicitado aos voluntários levar o objeto próximo à boca ou face, observando a atividade EMG do músculo bíceps braquial. Por fim, os voluntários deveriam retornar o objeto à mesa, observando e controlando a atividade do músculo tríceps braquial. Os objetos utilizados foram copos e bolas pequenas de diferentes materiais e pesos.

Após completar todo o treinamento, os pacientes foram reavaliados pelo mesmo fisioterapeuta. Esse fisioterapeuta ficou responsável somente pelas avaliações, não participando dos programas de reabilitação de ambos os grupos.

\section{Análise Estatística}

As análises estatísticas foram realizadas no programa SPSS versão 16.0. Antes da análise de cada variável, a normalidade na distribuição dos dados foi verificada por meio do teste de Shapiro-Wilk. Por se tratar de um estudo com amostra reduzida, optou-se pelo uso de testes não-paramétricos. As comparações intergrupos dos dados antropométricos e demográficos foram avaliadas com o teste Mann-Whitney $U$ (dados ordinais), e teste Qui-Quadrado (dados nominais). Para as comparações pré e pós-treinamento dos escores registrados nos testes clínicos foi utilizado o teste de Wilcoxon (Fugl-Meyer, TEMPA e Ashworth). Já a análise inter-grupos foi realizada por meio do teste Mann-Whitney $U$. Em todas as situações foi utilizado um nível de significância de $p \leq .05$.

\section{RESULTADOS}

Quatorze pacientes foram incluídos no presente estudo. No entanto, dois pacientes, sendo um do grupo fisioterapia e outro do grupo feedback EMG desistiram do estudo durante o período de treinamento. Nenhuma diferença estatisticamente significativa foi encontrada entre os grupos com respeito à idade, sexo, tempo de AVE, escores dos testes aplicados na avaliação funcional pré-treinamento. Entretanto, o grupo 2 apresentou valores maiores de índice de massa corpórea, em comparação ao grupo 1 (Tabela 1).

Na tabela 2 são apresentados a comparação intra-grupo dos escores obtidos na primeira avaliação (pré-treinamento) e segunda avaliação (pós-treinamento) no teste Fugl-Meyer e escala modificada de Ashworth. Foi possível observar que ambos os grupos apresentaram melhora na função motora do membro superior, visto que ocorreu aumento dos escores do teste Fugl-Meyer nos pacientes do grupo $1(p=.03)$ e do grupo $2(p=.03)$. Em relação ao quadro de espasticidade muscular, apesar da tendência de diminuição, não houve diferença estatística nos escores da escala modificada de Ashworth $(p \geq .12)$. Além disso, não foram observadas diferenças estatísticas nas comparações inter-grupos pré e pós-treinamento $(p \geq .58)$

Os resultados do teste TEMPA demonstraram que grupo de pacientes submetidos à fisioterapia convencional não apresentou evolução, enquanto que o grupo de pacientes submetido ao protocolo de treinamento com feedback EMG apresentou aumento nos escores de cinco tarefas e também a diminuição no tempo de execução de três tarefas, indicando assim melhora da funcionalidade em seis das oito tarefas avaliadas. Além disso, foi possível observar no grupo 2 uma melhora significativa do escore total e do tempo total de execução das tarefas (Tabela 3).

No entanto, a análise inter-grupos demonstrou que não houve diferença estatística nos ganhos médios dos escores do teste Fugl-Meyer $(p=.31)$ e do teste de Ashworth $(p \geq .37)$. Em relação aos ganhos médios dos escores do teste TEMPA, a análise estatística revelou que somente nas tarefas 5 $(p=.03)$ e $8(p=.04)$ foi possível observar maior eficiência do programa de reabilitação com feedback EMG. Nas demais tarefas e na soma total do TEMPA não foram observadas diferenças significativas inter-grupos $(p \geq .38)$ (Tabela 4$)$. 
Tabela 1

Características demográficas, antropométricas e escores iniciais do testes clínicos dos pacientes

\begin{tabular}{ccccc}
\hline & Grupo $1(n=6)$ & Grupo $2(n=6)$ & Teste & $P$ \\
\hline Idade & $52.67 \pm 17.84$ & $51.33 \pm 11.45$ & $U$ & .70 \\
Meses após AVE & $19.00 \pm 11.01$ & $16.50 \pm 10.31$ & $U$ & .63 \\
Sexo & 5 & 5 & $X^{2}$ & .99 \\
Homem & 1 & 1 & $X^{2}$ & .99 \\
Mulher & $23.17 \pm 1.45$ & $26.04 \pm 1.98$ & $U$ & $.03^{*}$ \\
Índice de Massa Corpórea (IMC) & $37.00 \pm 8.22$ & $31.00 \pm 9.12$ & $U$ & .27 \\
Fugl-Meyer (0-66) & & & & .81 \\
Ashworth & $2.17 \pm 0.41$ & $2.00 \pm 0.89$ & $U$ & .29 \\
Cotovelo (0-5) & $1.67 \pm 0.52$ & $2.17 \pm 0.75$ & $U$ & .70 \\
Punho (0-5) & $57.50 \pm 31.46$ & $66.66 \pm 28.27$ & $U$ & \\
TEMPA (0-186) & & & & \\
\hline
\end{tabular}

Os valores apresentados são média \pm desvio padrão.

Abreviações: U - Mann-Whitney e $\mathrm{X}^{2}$ - Qui-quadrado.

Tabela 2

Valores médios dos escores obtidos nos testes clínicos Fugl-Meyer e Ashworth em ambos os grupos

\begin{tabular}{ccccccc}
\hline & \multicolumn{2}{c}{ Fugl-Meyer } & \multicolumn{2}{c}{ Ashworth (Cotovelo) } & \multicolumn{2}{c}{ Ashworth (Punho) } \\
\hline & Grupo 1 & Grupo 2 & Grupo 1 & Grupo 2 & Grupo 1 & Grupo 2 \\
\cline { 2 - 7 } Avaliação1 & $37.00 \pm 8.22^{*}$ & $31.00 \pm 9.12^{*}$ & $2.17 \pm 0.41$ & $2.00 \pm 0.89$ & $1.67 \pm 0.52$ & $2.17 \pm 0.75$ \\
Avaliação2 & $47.83 \pm 13.78^{*}$ & $44.83 \pm 7.52^{*}$ & $1.50 \pm 0.55$ & $1.67 \pm 0.52$ & $1.50 \pm 0.55$ & $1.67 \pm 0.82$ \\
$P$ & .03 & .03 & .12 & .25 & .90 & .25 \\
\hline
\end{tabular}

Os valores apresentados são média \pm desvio padrão.

*Indica diferença estatística entre as avaliações 1 e 2

\section{DISCUSSÃO}

O presente estudo teve como objetivo avaliar e comparar o efeito de dois diferentes programas de treinamento (Feedback EMG X Fisioterapia convencional) para a reabilitação física de pacientes com sequelas motoras no membro superior em consequência ao AVE.

Os valores inicias do teste de Fugl-Meyer revelaram que os voluntários de ambos os grupos apresentavam comprometimento motor do membro superior classificado entre moderado e grave, com valores variando entre 23 e 44 pontos (Michaelsen \& Levin, 2004; Michaelsen et al., 2006). Após os treinamentos, foi possível confirmar que ambas as terapias aplicadas foram eficazes para a recuperação da função motora do membro superior, visto que ocorreu um aumento médio nos escores da escala de FMA de aproximadamente 10 pontos no grupo submetido ao protocolo de fisioterapia convencional e 14 pontos no grupo treinado com feedback EMG.
O aumento nos valores absolutos do teste FMA além serem considerados estatisticamente significativos, revelam que o grau do comprometimento motor do membro superior dos voluntários até então considerados entre moderado e grave, com os treinamentos, evoluíram para moderado a leve (valores variando entre 30 e 58 pontos) segundo escala descrita por Michaelsen e Levin (2004). Além disso, uma análise com valores relativos indicaram aumentos superiores a $30 \%$ nos escores do teste FMA, comprovando a melhora da função motora do membro superior, pois mudanças superiores a $10 \%$ são consideradas clinicamente importantes (Gladstone, Danells, \& Black, 2002; Stein, Narendran, McBean, Krebs, \& Hughes, 2007; Van der Lee et al., 1999). Na análise inter-grupos, nenhuma diferença estatística foi encontrada nos ganhos dos escores do teste FMA, demonstrando até então, evoluções similares na função motora do membro superior. 
Tabela 3

Valores médios dos escores obtidos no teste TEMPA para ambos os grupos

\begin{tabular}{|c|c|c|c|c|c|c|}
\hline & \multicolumn{3}{|c|}{ Grupo 1} & \multicolumn{3}{|c|}{ Grupo 2} \\
\hline & Avaliação 1 & Avaliação 2 & $P$ & Avaliação 1 & Avaliação 2 & $P$ \\
\hline Tarefa 1 & & & & & & \\
\hline Escore & $8.50 \pm 5.43$ & $6.17 \pm 4.92$ & .06 & $8.83 \pm 4.31$ & $5.17 \pm 5.34$ & $.01 *$ \\
\hline $\begin{array}{c}\text { Tempo (s) } \\
\text { Tarefa } 2\end{array}$ & $21.95 \pm 7.56$ & $26.78 \pm 14.10$ & .93 & $23.67 \pm 11.12$ & $20.10 \pm 11.50$ & $.01^{*}$ \\
\hline Escore & $9.00 \pm 3.03$ & $6.83 \pm 5.23$ & .16 & $9.83 \pm 2.93$ & $6.67 \pm 4.55$ & $.02 *$ \\
\hline $\begin{array}{c}\text { Tempo (s) } \\
\text { Tarefa } 3\end{array}$ & $43.87 \pm 18.71$ & $32.02 \pm 11.73$ & .06 & $39.90 \pm 18.22$ & $30.75 \pm 16.10$ & .09 \\
\hline Escore & $7.00 \pm 2.00$ & $5.67 \pm 3.67$ & .22 & $7.50 \pm 2.81$ & $5.67 \pm 4.03$ & .15 \\
\hline $\begin{array}{c}\text { Tempo (s) } \\
\text { Tarefa } 4\end{array}$ & $43.12 \pm 21.99$ & $30.66 \pm 17.17$ & .14 & $33.88 \pm 6.67$ & $31.49 \pm 9.20$ & .45 \\
\hline Escore & $6.83 \pm 3.31$ & $5.33 \pm 4.18$ & .06 & $6.83 \pm 2.78$ & $4.16 \pm 3.87$ & .09 \\
\hline $\begin{array}{c}\text { Tempo (s) } \\
\text { Tarefa } 5\end{array}$ & $54.03 \pm 17.04$ & $46.99 \pm 19.32$ & .34 & $50.49 \pm 16.18$ & $42.27 \pm 19.89$ & $.03 *$ \\
\hline Escore & $5.50 \pm 5.65$ & $4.50 \pm 6.44$ & .25 & $7.83 \pm 5.04$ & $3.83 \pm 4.83$ & $.01^{*}$ \\
\hline $\begin{array}{c}\text { Tempo (s) } \\
\text { Tarefa } 6\end{array}$ & $4.72 \pm 1.33$ & $4.06 \pm 1.26$ & .11 & $5.16 \pm 1.50$ & $3.60 \pm 1.51$ & $.01^{*}$ \\
\hline Escore & $6.67 \pm 4.55$ & $4.83 \pm 6.55$ & .19 & $10.00 \pm 4.90$ & $4.83 \pm 5.34$ & $.04 *$ \\
\hline $\begin{array}{c}\text { Tempo (s) } \\
\text { Tarefa } 7\end{array}$ & $20.92 \pm 7.03$ & $16.19 \pm 3.30$ & .11 & $20.09 \pm 7.54$ & $16.42 \pm 8.20$ & .07 \\
\hline Escore & $7.67 \pm 4.41$ & $6.17 \pm 5.46$ & .26 & $8.00 \pm 4.10$ & $5.17 \pm 5.64$ & $.02 *$ \\
\hline $\begin{array}{c}\text { Tempo (s) } \\
\text { Tarefa } 8\end{array}$ & $28.50 \pm 13.89$ & $21.55 \pm 16.21$ & .19 & $25.35 \pm 19.14$ & $28.14 \pm 20.56$ & .75 \\
\hline Escore & $6.33 \pm 4.89$ & $5.83 \pm 6.05$ & .62 & $7.83 \pm 3.97$ & $4.17 \pm 5.56$ & .06 \\
\hline $\begin{array}{c}\text { Tempo (s) } \\
\text { Total }\end{array}$ & $20.04 \pm 9.87$ & $14.18 \pm 7.11$ & .06 & $25.73 \pm 17.50$ & $11.66 \pm 5.72$ & .07 \\
\hline Escore & $57.50 \pm 31.46$ & $45.33 \pm 41.81$ & .10 & $66.67 \pm 28.27$ & $46.33 \pm 34.05$ & $.03 *$ \\
\hline Tempo (s) & $221.14 \pm 64.00$ & $183.00 \pm 50.85$ & .22 & $215.97 \pm 45.74$ & $174.54 \pm 55.66$ & $.03 *$ \\
\hline
\end{tabular}

Os valores apresentados são média \pm desvio padrão.

Os valores de escore representam a soma entre a graduação funcional e a análise das tarefas.

O tempo está apresentado em segundos (s).

*Indica diferença estatística entre as avaliações 1 e 2.

Tabela 4

Valores médios dos ganhos obtidos (Avaliação 2 - Avaliação 1), nos teste avaliados, em ambos os grupos

\begin{tabular}{cccc}
\hline & Grupo 1 & Grupo 2 & $p$ \\
\hline $\begin{array}{c}\text { Fugl-Meyer } \\
\text { Ashworth } \\
\text { (cotovelo) }\end{array}$ & $-0.66 \pm 0.51$ & $-0.50 \pm 0.54$ & .68 \\
Ashworth & $-0.16 \pm 0.40$ & $-0.50 \pm 0.54$ & .37 \\
(punho) & -- & -- & -- \\
TEMPA & $-2.33 \pm 2.33$ & $-2.00 \pm 1.79$ & .98 \\
Tarefa 1 & $-2.16 \pm 3.25$ & $-2.16 \pm 1.72$ & .87 \\
Tarefa 2 & $-1.33 \pm 2.33$ & $-0.83 \pm 1.33$ & .87 \\
Tarefa 3 & $-1.50 \pm 1.37$ & $-1.50 \pm 1.76$ & .99 \\
Tarefa 4 & $-1.00 \pm 1.09 *$ & $-4.66 \pm 3.07 *$ & .03 \\
Tarefa 5 & $-1.83 \pm 2.56$ & $-3.33 \pm 1.86$ & .38 \\
Tarefa 6 & $-1.50 \pm 2.25$ & $-2.00 \pm 1.26$ & .57 \\
Tarefa 7 & $-0.50 \pm 2.06^{*}$ & $-2.83 \pm 1.59 *$ & .04 \\
Tarefa 8 & $-12.16 \pm 13.67$ & $-18.66 \pm 9.16$ & .57 \\
Total & -13.67
\end{tabular}

Os valores apresentados são média \pm desvio padrão.

*Indica diferença estatística entre os grupos 1 e 2.

Ao se avaliar a função motora do membro superior com ênfase em aspectos como funcionalidade e destreza manual, os resultados do teste TEMPA revelaram que o grupo submetido à técnica de feedback EMG obteve evolução significativa em seis das oito tarefas testadas e também no escore total. O grupo submetido à fisioterapia convencional não apresentou diferenças significativas pós-treinamento nos escores do teste TEMPA.

Em uma análise inicial é possível observar que o treinamento com o feedback EMG proporcionou resultados positivos na melhora da função motora do membro superior, visto que foi identificado melhora dos escores de seis tarefas e do escore total. Entretanto, ao 
comparar a média dos ganhos obtidos no teste TEMPA, se observou maiores ganhos do grupo feedback EMG em apenas duas tarefas (tarefas 5 e 8), não existindo diferenças entre os grupos no escore total e nas demais tarefas.

Diferente do valores do FMA, não se encontra descrito na literatura, para o teste TEMPA aplicado à pacientes com AVE, valores de referência que indicam melhora clínica. No entanto, considerando que estudo prévio (Michaelsen et al., 2008) demonstrou que os resultados do teste TEMPA tem alta correlação com os resultados do FMA quando aplicados à pacientes com AVE, é possível considerar que ganhos superiores à $10 \%$ no TEMPA também sejam clinicamente significativos. No presente estudo o grupo fisioterapia apresentou evolução média de $20 \%$ no escore total, enquanto que o grupo feedback EMG apresentou 30 \%. Esses resultados sugerem que ambas as técnicas foram eficazes, do ponto de vista clínico, na reabilitação motora do membro superior.

As possíveis explicações para os diferentes resultados das comparações intra e inter-grupos podem estar relacionadas à especificidade do treinamento e ao nível da função motora dos voluntários no pré-treinamento. Com relação à especificidade, o fato do grupo feedback EMG ter realizado por sessão cerca de 25 minutos de treino das tarefas de alcance e preensão, atividades essas incluídas nas oito tarefas do teste TEMPA, pode explicar o melhor desempenho dos voluntários desse grupo em relação ao grupo fisioterapia. Os voluntários do grupo fisioterapia praticaram por sessão aproximadamente 20 minutos de treinamento de tarefas de auto-cuidado, alcance e preensão, sendo esse tempo dividido igualmente para cada tarefa, o que totalizou um tempo aproximado de 15 minutos para a tarefa de alcance e preensão.

Considerando a diferença de 10 minutos entre os grupos em cada sessão, é possível observar que o grupo feedback EMG obteve ao final do treinamento, seis horas a mais de treino de atividades de alcance e preensão. Esse fato corrobora os resultados de Platz et al. (2001) e Platz et al. (2009) que demonstraram que além da intensidade, o fator mais importante para o ganho da destreza manual é a especificidade do treinamento.

Outro aspecto importante que pode ter contribuído para os resultados entre as análises intra e intergrupos, principalmente no que diz respeito as diferenças ínfimas que foram observadas nos ganhos de cada grupo, é o nível da função motora dos voluntários no pré-treinamento. Embora não tenha sido observada diferença estatística dos valores iniciais do FMA entre os dois grupos, é importante considerar que existia uma diferença de aproximadamente $20 \%$ nos valores médios do FMA, sendo esta clinicamente relevante.

Michaelsen, Dannenbaum e Mindy (2006) demonstraram que pacientes crônicos com maior nível de comprometimento motor do membro superior, quando submetidos à treinos de tarefas específicas e funcionais apresentam maiores ganhos funcionais, em comparação aos indivíduos com comprometimento motor leve. Esse achado corrobora os resultados do presente estudo, no qual foram observados ganhos significativos do grupo feedback EMG, grupo esse que apresentou menor nível inicial da função motora do membro superior.

Apesar de existir na literatura científica um considerável número de estudos que avaliam os benefícios do uso do feedback EMG na reabilitação de pacientes com hemiparesia, observa-se uma grande dificuldade para a comparação entre os resultados, pois grande parte dos trabalhos têm utilizado o feedback EMG como ferramenta para treino da marcha (Aiello et al., 2005; Bradley et al., 1998; Jonsdottir et al., 2007). Além disso, os estudos que avaliam a eficácia do uso do feedback EMG na reabilitação do membro superior em pacientes com hemiparesia são em menor número, mais antigos (Armagam, Tascioglu, \& Oner, 2003; Basmajian, Gowland, Brandstater, Swanson, \& Trotter, 1982; Crow et al., 1989; Hemmen \& Seelen, 
2007; Hurd et al., 1980; Inglis et al., 1984) e tem utilizado diferentes ferramentas e escalas de avaliação, o que segundo Woodford e Price (2007) dificulta comparações com outras técnicas e conclusões sobre sua superioridade.

Estudos prévios (Hurd et al., 1980; Inglis et al., 1984) sugerem que o treinamento com feedback EMG associado à fisioterapia apresenta melhores resultados que a fisioterapia realizada isoladamente. Divergindo desses resultados, vários estudos demonstraram não existir superioridade da técnica de feedback EMG em relação à fisioterapia na reabilitação do membro superior em pacientes com hemiparesia (Lee, Hill, Johnston, \& Smiehorowski, 1976; Mroczek, Halpern, \& McHugh, 1978; Wolf et al., 1994). Entretanto, a função motora dos pacientes, de todos estudos supracitados, foi apenas avaliada por meio da atividade eletromiográfica de músculos superficiais e pela análise da amplitude de movimento articular (ADM) do membro superior, limitando qualquer conclusão sobre esses resultados. Principalmente, porque a função motora não pode ser resumida somente na ADM, sendo influenciada por outros vários aspectos não avaliados, inviabilizando assim a discussão e comparação com os resultados do presente estudo.

Basmajian et al. (1987), após quinze sessões de treinamento, assim como Armagam et al. (2003) após vinte sessões de treinamento, observaram resultados similares entre o grupo de pacientes submetidos à fisioterapia convencional e grupo que realizou o tratamento com a associação da fisioterapia e o feedback EMG, tanto para as escalas de análise de comprometimento motor, corroborando com os resultados do presente estudo; quanto para os testes funcionais, o que diverge dos nossos resultados. Em divergência, Crow et al. (1989) com base nos resultados encontrados após vinte sessões de treinamento, sugeriu em seu estudo que a associação da fisioterapia ao feedback EMG proporcionou resultados superiores no teste de FMA do grupo que recebeu o protocolo conven- cional de reabilitação.

Como possíveis explicações para as divergências encontradas entre o presente estudo e os estudos supracitados, estão especialmente as diferenças metodológicas. Primeiramente, os estudos prévios compararam a fisioterapia convencional e a sua associação com o feedback EMG em treinamentos que variaram entre 15 e 20 sessões com duração média de 30 minutos, enquanto que no presente estudo foram avaliadas as técnicas separadamente por número de sessões maior e com duração de 50 minutos para cada técnica. Esses fatores podem explicar o melhor desempenho, pois é demonstrado na literatura que a realização de tarefas específicas repetidas vezes, por períodos mais prolongados e de forma regular, é considerada fator determinante para a facilitação da reorganização cortical, com concomitante aumento da habilidade motora e melhora do desempenho das atividades funcionais (Liepert, Uhde, Graf, Leidner, \& Weiller, 2001).

Outro fator importante que pode ter colaborado para os diferentes resultados, foi a seleção e inclusão dos voluntários nos estudos. Os estudos prévios (Armagam et al., 2003; Basmajian et al., 1982; Basmajian et al., 1987; Crow et al., 1989) incluíram pacientes com hemiparesia nos lados direito ou esquerdo sem considerar a dominância funcional, diferentemente, o presente estudo incluiu somente pacientes destros com hemiparesia a direita, ou seja, somente foram incluídos pacientes com comprometimento do lado dominante. O comprometimento de diferentes hemisférios cerebrais podem causar não somente características comportamentais e intelectuais distintas, mas também podem influenciar no processo de recuperação da função motora. Pacientes com lesão no hemisfério direito, podem apresentar dificuldade com início, sequência e direção do movimento, enquanto pacientes com lesão do hemisfério esquerdo tem tendência a apresentar distúrbios visuais, diminuição da atenção e dificuldades com discriminação de tamanho 
e distâncias de objetos (Yekutiel \& Guttman, 1993).

Byl et al (2003) compararam a evolução da função motora de pacientes com hemiparesia após serem submetidos a um protocolo de reabilitação embasado nos princípios da neuroplasticidade, os autores observaram que não houve diferença no controle motor fino e na performance muscular entre pacientes com hemiparesia a direita e a esquerda. No entanto, os pacientes com hemiparesia à direita apresentaram ganhos significativos comparados aos pacientes com hemiparesia à esquerda, em relação a velocidade da marcha, discriminação sensorial e principalmente na independência funcional. Além disso, o comprometimento do lado dominante, pode ter proporcionado uma maior motivação nos pacientes, afim de recuperar o lado afetado para realização das atividades de vida diária.

Os valores encontrados por meio da escala modificada de Ashworth revelaram uma tendência de diminuição da espasticidade na comparação pré e pós-treinamento. Contudo, nenhuma diferença significativa foi observada entre os voluntários submetidos aos dois tipos de treinamento, concordando com resultados prévios (Bourbonnais et al., 2002; Stein et al., 2007). Assim como na avaliação intra-grupo, a comparação inter-grupos mostrou não existir diferenças estatísticas nas avaliações pré e pós-treinamento, sugerindo que a espasticidade não foi fator predisponente à melhora de um grupo em relação ao outro.

Apesar de não ser observado diferença estatística, a tendência à diminuição da espasticidade pode ter influenciado positivamente a melhora funcional dos voluntários de ambos os grupos. Esses resultados corroboram as observações feitas por outros pesquisadores (Fasoli et al., 2004), de que treinamentos funcionais combinados com aplicação de tarefas resistidas seja de forma manual ou mecânica, não fazem com que exacerbe a espasticidade em pacientes com hemiparesia decorrente do AVE.
Mais estudos são necessários para compreender os diferentes mecanismos e aspectos envolvidos no processo de reabilitação de pacientes hemiparéticos. Devem ser realizados novos estudos clínicos com amostras maiores, pois assim será possível obter evidências sobre os benefícios e a viabilidade da inserção e associação do feedback EMG nos programas convencionais de reabilitação dos pacientes vítimas do AVE.

\section{CONCLUSÕES}

Os resultados do presente estudo mostraram que treinamento de tarefas funcionais associado ao feedback EMG se mostrou uma técnica viável e que pode apresentar resultados positivos na melhora da função motora do membro superior. Por outro lado, considerando os ganhos, do ponto de vista estatístico e clínico, foi possível observar que ambas as técnicas são eficazes na reabilitação motora do membro superior, não existindo evidências de superioridade de uma técnica em relação à outra.

Agradecimentos:

Nada declarado.

Conflito de Interesses:

Nada declarado.

Financiamento:

Nada declarado.

\section{REFERÊNCIAS}

Aiello, E., Gates, D. H., Patritti, B. L., Cairns, K. D., Meister, M., Clancy, E. A. et al. (2005). Visual EMG feedback to improve ankle function in hemiparetic gait. Conference Proceedings IEEE Enginnering in Medicine and Biology, 7, 7703-7706. doi: 10.1109/IEMBS.2005.1616297

Armagan, O., Tascioglu, F., \& Oner, C. (2003). Electromyographic feedback in the treatment of the hemiplegic hand: a placebo-controlled study. Archives of Physical Medicine Rehabilitation, 82 (11), 856-861. 
Basmajian, J. V., Gowland, C. A., Brandstater, M. E., Swanson, L., \& Trotter, J. (1982). EMG feedback treatment of upper limb in hemiplegic stroke patients: a pilot study. Archives of Physical Medicine Rehabilitaton, 63(12), 613-616.

Basmajian J. V., Gowland, C. A., Finlayson, M. A., Hall, A. L., Swanson, L. R., Stratford P. W. et al. (1987). Stroke treatment: comparison of integrated behavioral-physical therapy vs traditional physical therapy programs. Archives of Physical Medicine Rehabilitaton, 68(5), 267-272.

Bourbonnais, D., Bilodeau, S., Lepage, Y., Beaudoin, N., Gravel, D., \& Forget, R. (2002). Effect of force-feedback treatments in patients with chronic motor deficits after a stroke. American Journal of Physical Medicine Rehabilitation, 81 (12), 890-897.

Bradley, L., Hart, B. B., Mandana, S., Flowers, K., Riches, M., \& Sanderson, P. (1998). Electromyographic feedback for gait training after stroke. Clinical Rehabilitation, 12(1), 11-22. doi: 10.1191/026921598677671932

Brashear, A., Zafonte, R., Corcoran, M., Galvez-jimenez, N., Gracies, J. M., Gordon, M. F. et al. (2002). Inter- and intrarater reliability of the Ashworth Scale and the Disability Assessment Scale in patients with upper-limb poststroke spasticity. Archives of Physical Medicine Rehabilitation, 83(10), 1349-1354. doi:10.1053/ apmr.2002.35474

Byl, N., Roderick, J., Mohamed, O., Hanny, M., Kotler, J., Smith, A. et al. (2003). Effectiveness of Sensory and Motor Rehabilitation of the Upper Limb Following the Principles of Neuroplasticity: Patients Stable Poststroke. Neurorehabilitation Neural Repair, 17(3), 176-91. doi: 10.1177/0888439003257137

Crow, J. L., Lincoln, N. B., Nouri, F. M., \& Weerdt, W. (1989). The effectiveness of EMG feedback in the treatment of arm function after stroke. International disability studies, 11(4), 155-160.

Fasoli, S. E., Krebs, H. I., Stein, J., Frontera, W. R., Hughes, R., \& Hogan, N. (2004). Robotic therapy for chronic motor recorvery after stroke: Follow-up results. Archives of Physical Medicine
Rehabilitation, 85(7), 1106-11. doi:10.1016/j. apmr.2003.11.028

Feigin, V. L. (2005). Stroke epidemiology in the developing world. Lancet, 365(9478), 2160-1. doi:10.1016/S0140-6736(05)66755-4.

Fugl-Meyer, A. R., Jaasko, L., Leyman, I., Olsson, S., \& Steglind S. (1975). The post-stroke hemiplegic patient. 1. a method for evaluation of physical performance. Scandinavian Journal of Rehabiitationl Medicine, 7(1), 13-31.

Gladstone, D. J., Danells C. L., \& Black, S. E. (2002). The Fugl-Meyer Assessment of Motor Recovery after Stroke: A Critical Review of Its Measurement Properties. Neurorehabilitaton Neural Repair, 16(3), 232-40. doi: $10.1177 / 154596802401105171$

Gowland, C. (1982). Recovery of motor function following stroke: profile and predictors. Physiotherapy, 34, 77-84.

Hermes, H. J., Freriks, B., Merletti, R., Stegeman, D., Blok, J., Rau, G. et al. (1999). European Recommendations for Surface Electromyography - Results of the SENIAN project. Enschede, Netherlands: Roessingh Research and Development.

Hu, X. L., Tong, X. L., Song, R., Zheng, X. J., Lui, K. H., Leung, K. H. et al. (2009). Quantitative evaluation of motor functional recovery process in chronic stroke patients during robot-assisted wrist training. Journal of Electromyography and Kinesiology, 19(4), 639-650. doi:10.1016/j. jelekin.2008.04.002

Hurd, W. W., Pegram, V., \& Nepomuceno, C. (1980). Comparison of actual and simulated EMG feedback in the treatment of hemiplegic patients. American Journal of Physical Medicine, 59(2), 73-82.

Hurrel, M. (1980). Electromyographic feedback in rehabilitation. Physiotherapy, 66(9), 293-298.

Inglis, J., Donald, M. W., Monga, T. N., Sproule, M., \& Young, M. J. (1984). Electromyographic feedback and physical therapy of the hemiplegic upper limb. Archives of Physical Medicine Rehabilitation, 65(12), 755-759.

Jonsdottir, J., Cattaneo, D., Recalcati, M., Regola, A., Rabuffetti, M., Ferrarin, M. et al. (2010). 
Task-oriented biofeedback to improve gait in individuals with chronic stroke: motor learning approach. Neurorehabilitation Neural Repair, 24(5), 478-85.

Jonsdottir, J., Cattaneo, A., Regola, A., Crippa, A., Recalcati, M., Rabuffetti, M. et al. (2007). Concepts of Motor Learning Applied to a Rehabilitation Protocol Using Feedback to Improve Gait in a Chronic Stroke Patient: An A-B System Study With Multiple Gait Analyses. Neurorehabilitation Neural Repair, 21(2), 190-94. doi: $10.1177 / 1545968306290823$

Kwakkel, G., Kollen, B. J., \& Wagenaar, R. C. (1999). Therapy impact on functional recovery in stroke rehabilitation: a critical review of the literature. Physiotherapy, 13, 457-70. doi:10.1016/S00319406(05)67198-2

Lee, K. H., Hill, E., Johnston, R., \& Smiehorowski, T. (1976). Myofeedback for muscle retraining in hemiplegic patients. Archives of Physical Medicine Rehabilitation, 57(12), 588-591.

Levy, C. E., Nichols, D. S., Schmalbrock, P. M., Keller, P., \& Chakeres D. W. (2001). Functional MRI evidence of cortical reorganization in upper-limb stroke hemiplegia treated with constraint-induced movement therapy. American Journal of Physical Medicine Rehabilitation, 80(1), 4-12.

Liepert, J., Bauder, H., Wolfgang, H. R., Miltner, W. H., Taub, E., \& Weiller, C. (2000). Treatment-induced cortical reorganization after stroke in humans. Stroke. 31 (6), 1210-6. doi: 10.1161/01. STR.31.6.1210

Liepert, L., Uhde, I., Graf, S., Leidner, O., \& Weiller, C. (2001). Motor cortex plasticity during forced-use therapy in stroke patients: a preliminary study. Journal of Neurology, 248(4), 315-321. doi: $10.1007 / \mathrm{s} 004150170207$

Michaelsen, S. M., \& Levin, M. F. (2004). Short-term effects of practice with trunk restraint on reaching movements in patients with chronic stroke: a controlled trial. Stroke, 35(8), 1914-1919. doi: 10.1161/01. STR.0000132569.33572.75

Michaelsen, S. M., Dannenbaum, R., \& Levin, M. F. (2006). Task-specific training with trunk restraint on arm recovery in stroke: randomized control trial. Stroke, 37(1), 186-192. doi: 10.1161/01.STR.0000196940.20446.c9v1

Michaelsen, S. M., Natalio, M., Silva, A. G., \& Pagnussat, A. S. (2008) Reliability of the translation and adaptation of the Test d'Évaluation des Membres Supérieurs des Personnes Âgées (TEMPA) to the Portuguese language and validation for adults with hemiparesis. Revista Brasileira de Fisioterapia, 12(6), 511-9. doi: 10.1590/ S1413-35552008005000012

Michaelsen, S. M., Rocha, A. S., Knabben, R. J., Rodrigues, L. P., \& Fernandes, C. G. (2011). Translation, adaptation and inter-rater reliability of the administration manual for the Fugl-Meyer assessment. Revista Brasileira de Fisioterapia, 15(1), 80-8. doi: 10.1590/S141335552011000100013

Moreland, J. D., Thomson, A. N. (1994). Efficacy of electromyographic biofeedback compared with conventional physical therapy for upper-extremity function in patients following stroke: A research overview and meta-analysis. Physical Therapy 74(6), 534-543.

Moreland, J. D., Thomson, A. N., \& Fuoco. A. R. (1998). Electromyographic feedback to improve lower extremity function after stroke: A meta-analysis. Archives of Physical Medicine Rehabilitation, 79(2), 134-40.

Mroczek, N., Halpern, D., \& McHugh, R. (1978). Electromyographic feedback and physical therapy for neuromuscular retraining in hemiplegia. Archives of Physical Medicine Rehabilitation, 59(6), 258-67.

Muntner, P., Garret, E., Klag, M. J., \& Coresh, J. (2002). Trends in stroke prevalence between 1973 and 1991 in the US population 25 to 74 years of age. Stroke, 33(5),1209-1213. doi: 10.1161/01.STR.0000015031.57955.D1

Platz, T., Van Kaick, S., Mehrholz, J., Leidner, O., Eickhof, C., Pohl, M. (2009). Best Conventional Therapy Versus Modular Impairment-Oriented Training for Arm Paresis After Stroke: A Single-Blind, Multicenter Randomized Controlled Trial. Neurorehabilitation and Neural Repair, 23(7), 
706-716. Doi: 10.1177/1545968309335974

Rathore, S. S., Hinn, A. R., Cooper, L. S., Tyroler, H. A., \& Rosamond, W. D. (2002). Characterization of incident stroke signs and symptoms: findings from the arthesclerosis risk in communities study. Stroke, 33(11), 2718-21. doi: 10.1161/01.STR.0000035286.87503.31

Schaechter, J. D., Kraft, E., Hilliard, T. S., Dijkhuizen, R. M., Benner, T., Finklestein et al. (2002). Motor recovery and cortical reorganization after constraint-induced movement therapy in stroke patients: a preliminary study. Neurorehabilitation Neural Repair. 16(4), 326-38. doi: 10.1177/154596830201600403

Stein, J., Narendran, K., McBean, J., Krebs, K., \& Hughes, R. (2007). Electromyography controlled exoskeletal upper-limb-powered orthosis for exercise training after stroke. American Journal of Physical Medicine Rehabilitation, 86(4), 255-261. doi: 10.1097/ PHM.0b013e3180383cc5
Van der Lee, J. H., Wagenaar, R. C., Lankhorst, G. J., Vogelaar, T. W., Devillé, W. L, \& Bouter, L. M. (1999). Forced use of the upper extremity in chronic stroke patients: Results from a single-blind randomized clinical trial. Stroke, 30(11), 2369-75. doi: 10.1161/01.STR.30.11.2369

Woodford H. J., \& Price, C. (2007). Electromyographic Feedback for the Recovery of Motor Function After Stroke. Stroke, 38(2), 1999-2000. doi: 10.1002/14651858.CD004585.pub2

Yekutiel, M., \& Guttman, E. (1993). A controlled trial of the retraining of the sensory function of the hand in stroke patients. Journal of neurology, neurosurgery, and psychiatry, 56(3), 241-4.

(cc) EY-NC Todo o conteúdo da revista Motricidade está licenciado sob a Creative Commons, exceto quando especificado em contrário e nos conteúdos retirados de outras fontes bibliográficas. 